\title{
EDUCAÇÃO SANITÁRIA COM FOCO NA SEGURANÇA DE ALIMENTOS: UMA ABORDAGEM PARA CRIANÇAS
}

\author{
Gustavo Nunes de Moraes ${ }^{1}$ \\ Teng Fwu Shing ${ }^{1}$ \\ Carolina Rosa Rodrigues de Souza ${ }^{1}$ \\ Giovanni Costa Danelon ${ }^{1}$ \\ Luciana Tiemi Hioka ${ }^{1}$ \\ Rafaela Teston Campanha ${ }^{1}$ \\ Maria Helena Vizzuso ${ }^{1}$ \\ Juliana Barbosa da Silva ${ }^{1}$ \\ Gabrielle dos Santos Rocha ${ }^{1}$ \\ Marise Santiago Velame ${ }^{2}$ \\ Juliano Gonçalves Pereira ${ }^{3}$
}

\begin{abstract}
RESUMO
As doenças transmitidas por alimentos (DTAs) são definidas como enfermidades infecciosas ou tóxicas causadas pelo consumo de alimentos ou água contaminados. Na atualidade, são consideradas um dos maiores problemas mundiais de saúde pública. Dentro do contexto das DTAs, a higiene pessoal e a manipulação inadequada de alimentos no ambiente doméstico têm sido apontadas como importantes fatores de risco para a ocorrência de surtos. Baseado nestas considerações, o presente projeto de extensão universitária foi desenvolvido no intuito de instruir crianças em idade escolar sobre a importância da higiene pessoal e da manipulação adequada de alimentos, de modo que estas disseminem a informação a seus pais e familiares. O projeto foi instituido em uma escola pública do município de Botucatu-SP e atingiu 491 crianças de 5 a 11 anos. As atividades foram aplicadas em três ações prioritárias, sendo elas: 1) Palestra introdutória lúdica e interativa; 2) Gincana com brincadeiras e atividades formativas práticas; 3) Entrega de certificados de participação e cartilha contendo informações e jogos com enfoque nos assuntos abordados, de modo a possibilitar a disseminação das informações para os familiares e amigos dos participantes. Os alunos demonstraram grande interesse pelo assunto, enfatizando a importância dessa abordagem nas escolas. Foi possível promover, portando, educação em saúde de forma prazerosa, de modo a evitar doenças relacionadas à precária higiene pessoal e à manipulação incorreta de alimentos.
\end{abstract}

Palavras-chave: educação em saúde, ensino fundamental, higiene de alimentos, saúde pública

\begin{abstract}
Foodborne illnesses are any infectious or toxic diseases resulting from the consumption of contaminated food or water. They are currently considered one of the top public health issues worldwide. Inadequate personal hygiene and food handling in the family environment have been identified as major risk factors for the occurrence of outbreaks. In view of this, the current research aimed to educate school-age children in terms of the importance of proper personal hygiene and food handling, so that these considerations are spread out to their

\footnotetext{
${ }^{1}$ Graduandos de Medicina Veterinária da FMVZ-UNESP- Botucatu. gustavonunesdemoraes@gmail.com

${ }^{2}$ Mestrando em Medicina Veterinária da FMVZ-UNESP Botucatu

${ }^{3}$ Docente da Faculdade de Medicina Veterinária e Zootecnia da Universidade Estadual Paulista "Júlio de Mesquita Filho" UNESP. *Correspondência.
} 
household. The present extension project was carried out at a municipal school in the city of Botucatu-SP and included 491 children ranging from 5 to 11 years old. The activities focused on three priority actions: 1) entertaining and interactive introductory lectures; 2) gymkhana with playful and educational activities; 3) provision of participation certificates and a brochure with informative games related to the topics previously discussed, so that the newly acquired knowledge could be spread out to the child's friends and relatives. The students demonstrated deep interest over this theme, which emphasizes the importance of the current approach in schools. Therefore, health education was pleasantly promoted, contributing to prevent diseases related to the lack of personal hygiene and improper food handling.

Keywords: elementary school, food safety, health education, public health

\section{RESUMEN}

Las enfermedades transmitidas por los alimentos (DTA) se definen como enfermedades infecciosas o tóxicas causadas por el consumo de alimentos o água contaminados. Hoy en día, se consideran uno de los mayores problemas de salud pública a nivel mundial. En el contexto de los DTA, la higiene personal y la manipulación inadecuada de los alimentos en el entorno doméstico se han identificado como un factor de riesgo importante para los brotes. A partir de estas consideraciones este proyecto de extensión universitária fue creado para instruir a los niños en edad escolar sobre la importancia de la higiene personal y el manejo adecuado de los alimentos, para que difundan información a sus padres y familiares. El proyecto de extensión univertirária se aplicó en una escuela municipal del municipio de Botucatu-SP y llegó a 491 niños de 5 a 11 años. Las actividades se aplicaron en tres acciones prioritarias, a saber: 1) Conferencia introductoria lúdica e interactiva; 2) Gincana de juegos y actividades de entrenamiento práctico y; 3) Entrega de certificados de participación y cartilla con información y juegos centrados en los temas tratados, con el fin de permitir la difusión de información a familiares y amigos de los participantes. Los estudiantes mostraron un gran interés por el tema, destacando la importancia de este enfoque en las escuelas. Se logró, por tanto, promover la educación en salud de forma amena, con el fin de evitar enfermedades relacionadas con la falta de higiene personal y la manipulación incorrecta de los alimentos.

Palabras clave: educación sanitaria, educación primaria, higiene alimentaria, salud pública.

\section{INTRODUÇÃO}

A cada ano, cerca de 600 milhões de pessoas no mundo adoecem devido à ingestão de alimentos contamidados, seja por patógenos ou substâncias químicas. Destas, 420 mil são ocorrências fatais, sendo as crianças, idosos e pessoas com doenças primárias os principais integrantes destes números (1).

Doenças Transmitidas por Alimentos (DTAs) são causadas pela ingestão de alimentos ou água contaminada. Possuem como etiologia agentes físicos, químicos ou biológicos, sendo os microrganismos os principais envolvidos. São doenças emergentes em muitos países, originando problemas tanto na área da saúde pública como no âmbito econômico (2).

No Brasil, foram notificados 6.809 surtos, 120.584 casos e 99 mortes relacionados à DTAs entre 2009 e 2018. Destes surtos, quase 40\% ocorreram no ambiente doméstico (3). Apesar dos números, os casos envolvendo intoxicação alimentar são subnotificados, uma vez que muito deles são negligenciados. Acredita-se, portanto, que a proporção deste problema seja ainda maior (4).

Moraes GN, Shing TF, Souza CRR, Danelon GC, Hioka LT, Campanha RT, et al. Educação sanitária com foco na segurança de alimentos: Uma abordagem para crianças. Vet. e Zootec. 2021; v28: 001-010 
Alguns estudos têm demonstrado que o ambiente doméstico é o principal local relacionado a surtos de DTAs. No Brasil, dos surtos notificados no intervalo de 2000 a 2017 , $36.4 \%$ ocorreram no ambiente doméstico (5). Já na Europa, cerca de um terço dos surtos identificados em 2011 ocorreram nas residências (6). É dentro de casa, portanto, onde as práticas inadequadas de manipulação, higiene e armanezamento de alimentos tornam-se um grande risco para a saúde das pessoas (7).

Dentre as formas de prevenção dessas doenças, conceitos básicos como lavagem correta das mãos, armazenamento e higienização adequada dos alimentos destacam-se como pontos chaves, principalmente quando o risco está relacionado ao domicílio (2).

A educação em saúde é ferramenta fundamental para a promoção da saúde na sociedade (8). A partir disso, um programa de saúde escolar tende a ser um dos investimentos mais econômicos que uma nação pode fazer em benefício às esferas da educaçao e da saúde (9).

A união entre vários setores para o trabalho conjuntos em favorecimento à saúde pública tem-se o nome de Saúde Única. Tanto no cotexto de segurança alimentar, como em qualquer outro âmbito, a multidisciplinaridade envolvida na execução de trabalhos que envolvem a esfera da saúde é de extrema valia, uma vez que o ser humano, os animais e o ecossistema compartilham dos mesmos microrganismos (10).

Neste sentido, torna-se importante a aplicação de medidas de educação sanitária para a população, uma vez que muitos desconhecem acerca de inocuidade e segurança alimentar. Além disso, a educação sanitária voltada às crianças caracteriza-se por ter grande impacto na educação de pais e familiares, uma vez que estas disseminam informações e agem como agentes de transformação social.

Acredita-se que a pedagogia escolar e a educação sanitária devem estar em associação, sendo essêncial tal abordagem principalmente para crianças, visto que apresentam uma maior maleabilidade psicológica para adquirirem novos hábitos e perpetuarem tais informações na vida adulta (11)

Crianças em idade escolar apresentam grande capacidade de aprendizado e memorização, já que é nesta fase que suas habilidades visuais e auditivas estão se aperfeiçoando em conjunto. Além disso, a capacidade de memória das crianças é ampliada quando relacionada a sentimentos. Atividades lúdicas, portanto, tornam o aprendizado uma experiência agradável (12).

Neste sentido, o objetivo deste trabalho é descrever a experiência e as metodologias de ensino vivenciadas no projeto de extensão universitária intitulado "Educação sanitária com foco na segurança de alimentos: uma abordagem para crianças", desenvolvido em uma escola municipal pública de ensino fundamental I do município de Botucatu-SP.

\section{METODOLOGIA}

\section{Desenvolvimento do projeto}

O projeto de extensão em questão surgiu da necessidade de instruir a população sobre assuntos de higiene pessoal e de manipulação de alimentos, no intuito de promover ação direta em saúde pública. As crianças foram o alvo deste estudo dado que, como dito anteriormente, apresentam capacidade de memória ampliada e grande poder de disseminar informações para seus familiares.

A equipe organizadora do projeto foi composta por nove alunos de graduação em Medicina Veterinária e dois Médicos Veterinários especializados em segurança dos aimentos, sendo um deles aluno de pós-graduação e o outro coordenador responsável pelo grupo. Estes fazem parte do Grupo de Estudos em Inspeção e Segurança Alimentar (GEISA), da Faculdade de Medicina Veterinária e Zootecnia da UNESP, câmpus de Botucatu-SP (FMVZ Botucatu).

Moraes GN, Shing TF, Souza CRR, Danelon GC, Hioka LT, Campanha RT, et al. Educação sanitária com foco na segurança de alimentos: Uma abordagem para crianças. Vet. e Zootec. 2021; v28: 001-010 
Além da equipe organizadora, o projeto contou com a participação voluntária de aproximadamente 40 alunos de graduação em Medicina Veterinária e Zootecnica e pósgraduandos da FMVZ.

Em abril de 2019, início do projeto, toda a equipe envolvida no trabalho foi submetida a uma reunião, no qual foi possível a discussão acerca dos objetivos almejados e das atividades que seriam aplicadas. A partir de então, o grupo se reuniu semanalmente para a construção e confecção das atividades propostas para o projeto. Uma vez finalizadas, foi elaborado um treinamento prático para correções e ajustes dos discursos e da organização durante a abordagem com as crianças.

Após a aprovação da Prefeitura de Botucatu e da Faculdade de origem do grupo (FMVZ Botucatu), as atividades foram efetivadas no próprio espaço escolar, entre os dias 11 e 15 de novembro de 2019, em período integral na Escola de ensino fundamental I Prof. Marins, em Botucatu-SP. A execução das atividades foi ao longo do dia letivo das crianças. As dinâmicas foram aplicadas por turmas, tendo duração de aproximadamente uma hora e meia em cada turma.

\section{Atividades desenvolvidas com as crianças}

Foram desenvolvidas três atividades principais: (1) Palestra interativa, (2) Gincana com atividades práticas e lúdicas (3) Entrega de material educativo impresso.

1. Palestra interativa: foi constituída de temas relacionados à higiene pessoal e de alimentos, tendo enfoques no processo de lavagem de mãos, no armazenamento correto de alimentos e nas diferenças entre microrganismos patogênicos e não patogênicos. Além disso, foram apresentadas formas de higienização de alimentos e como armazená-los corretamente. Todas as informações foram ilustrativas e de fácil entendimento, com uso de recursos de multimídia e interações entre os membros da equipe organizadora com as crianças (Figura 1). Esta foi uma atividade de abertura do projeto na escola, no qual permitiu um primeiro contado entre os alunos e os organizadores do projeto, possibilitanto uma maior relação de confiança entre eles. Posteriormente, os alunos foram convidados a participar das atividades práticas da gincana.

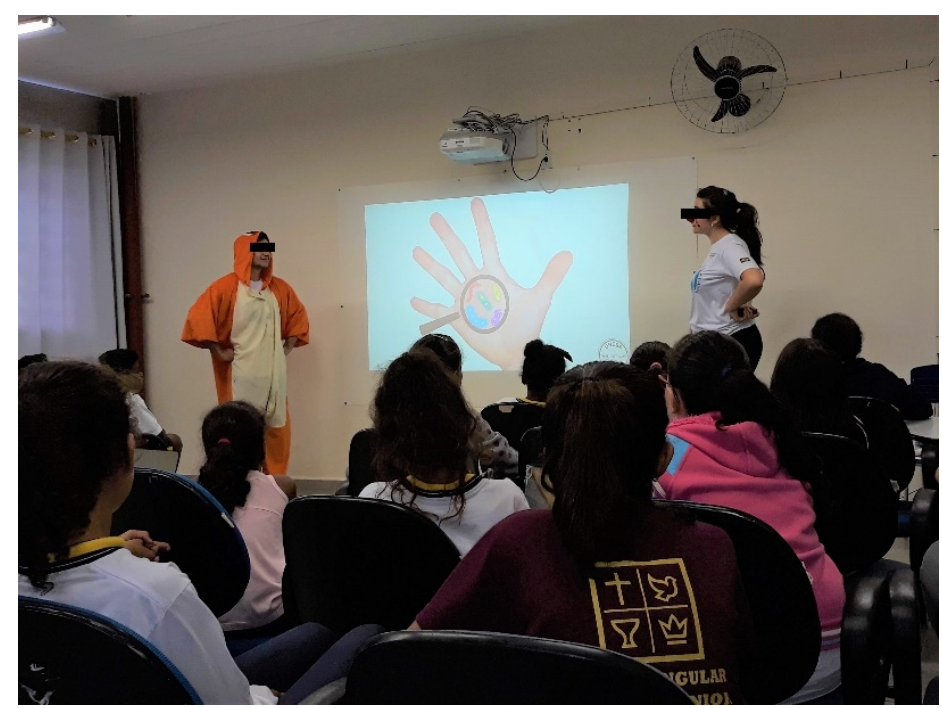

Figura 1. Apresentação da palestra inicial para os alunos participantes do projeto de extensão.

A palestra teve aproximadamente 20 minutos de duração para cada turma, e os assuntos abordados consistiram no seguinte roteiro:

- Vídeo adaptado do filme "Divertidamente", redublado, seguido pela pergunta: "Já

Moraes GN, Shing TF, Souza CRR, Danelon GC, Hioka LT, Campanha RT, et al. Educação sanitária com foco na segurança de alimentos: Uma abordagem para crianças. Vet. e Zootec. 2021; v28: 001-010 
olhou para alguém e pensou: o que passa na mão dela?";

- Foto da mão com bactérias animadas e explicação básica sobre microrganismos;

- Interação com perguntas como: "Existem apenas bactérias 'do mal'?";

- Mostrar algumas "bactérias do bem" e dar exemplos de produtos (iogurtes, queijos);

- Focar em bactérias do mal, explicando sobre a probabilidade de causarem doenças;

- Falar brevemente sobre a etiologia das DTAs;

- Ensinar como evitar as doenças causadas por bactérias: como armazenar os diferentes alimentos de forma correta na geladeira e como higienizá-los;

- Ensinar como lavar as mãos corretamente (música "lava uma mão lava a outra" de plano de fundo).

2. Gincana com atividades práticas e lúdicas: Esta atividade consistiu em uma série de brincadeiras elaboradas com o intuito de promover uma interação maior entre os alunos e a equipe do projeto. Foram abordados de maneira prática os mesmos assuntos já apresentados na palestra. A equipe do projeto participou de forma ativa junto com as crianças, incentivando-as a participarem. Cada turma se dividiu em duas equipes, porém, ao final, todos foram vencedores e ganharam certificados de participação (Figura 2).

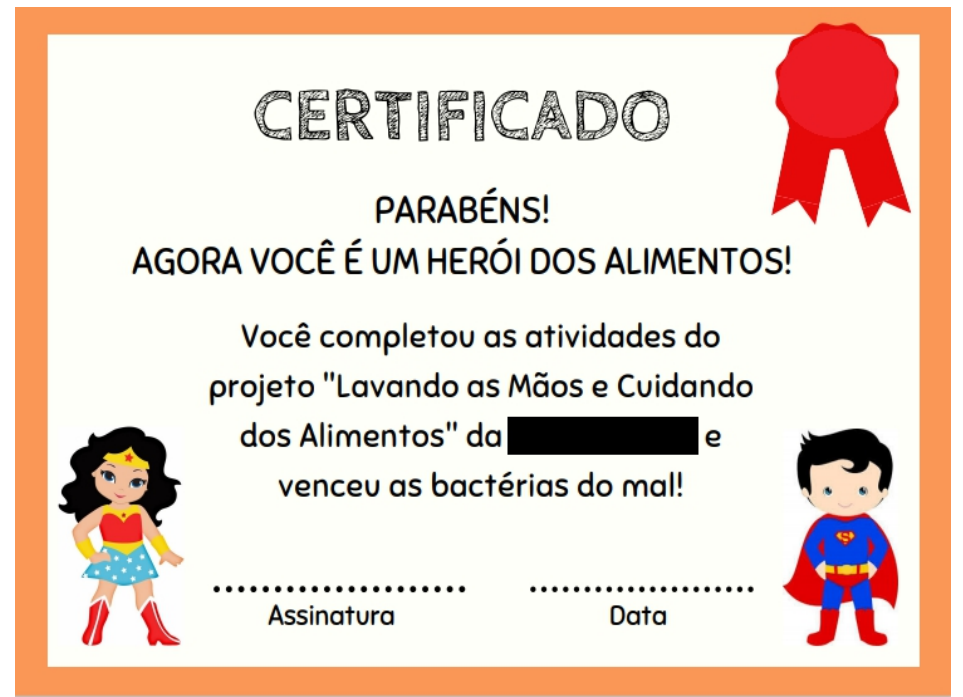

Figura 2. Certificado entregue para os alunos que participaram do projeto de extensão.

O tempo estimado da gincana foi de 30 minutos, divididos em três atividades:

- Posicionar alimentos na geladeira: Nesta atividade, foi posicionado um cartaz em formato de geladeira e figuras de alimentos que se aderem à superfície do cartaz. $O$ objetivo era que as crianças soubessem quais alimentos devem ir à geladeira e quais não precisam ser refrigerados (Figura 3). 


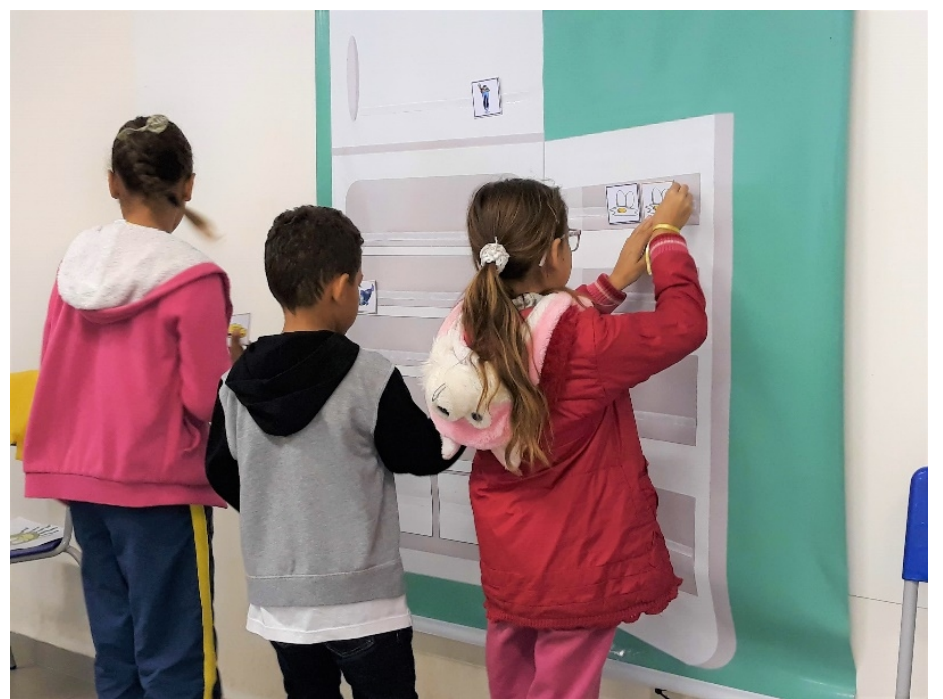

Figura 3. Realização da atividade "Posicionar alimentos na geladeira" durante a gincana.

- Jogo de associação: Foram apresentados slides contendo imagens de diversos alimentos e situações. As crianças deveriam, portanto, associá-los a microrganismos patogênicos ou não patogênicos (bactéria "do bem" versus bactéria "do mal"), representados por membros da equipe organizadora.

- Lavagem de mãos: Dois membros da organização do projeto foram vendados para simular a lavagem das mãos com o uso de tintas. As equipes das crianças ensinavam para os organizadores o passo a passo para a lavagem das mãos. A tinta iria dizer, portanto, o quão completa a lavagem estava (Figura 4).

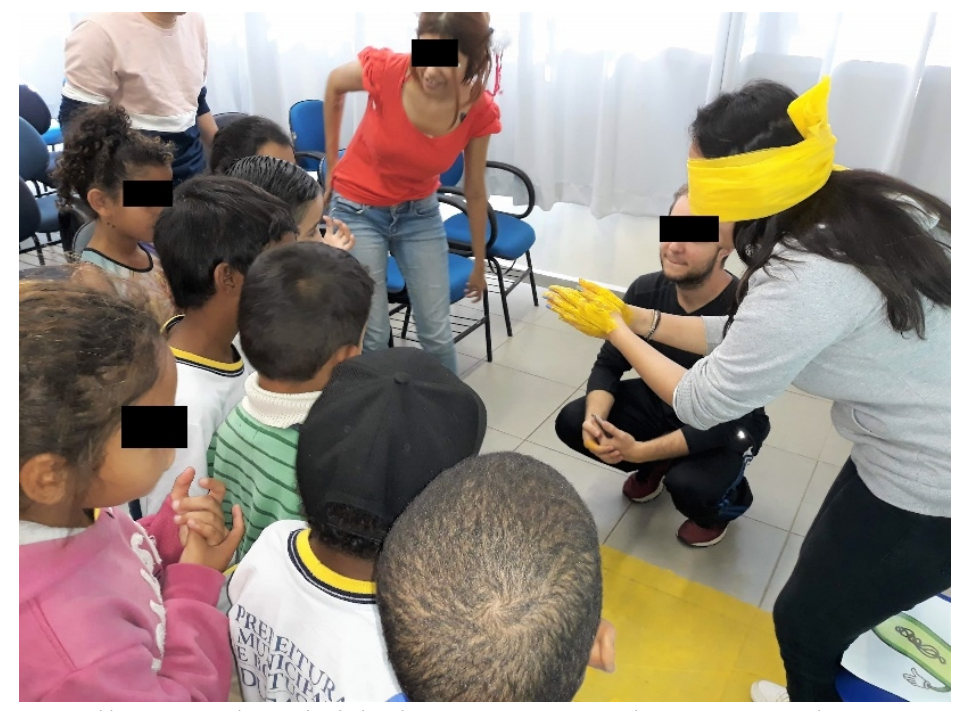

Figura 4. Realização da atividade "Lavagem das mãos" durante a gincana.

3. Material educativo impresso: $\mathrm{O}$ material em forma de cartilha apresentava atividades lúdicas e didáticas (caça-palavras, labirinto, desenho para colorir, ligue os pontos, etc) com temas relacionados àqueles já abordados. Este também apresentava pequenos textos informativos, com o objetivo de as crianças levarem tais informações adiante para seus familiares e amigos. A cartilha foi entregue ao final da gincana, funcionando como encerramento para as atividades do projeto (Figura 5). 


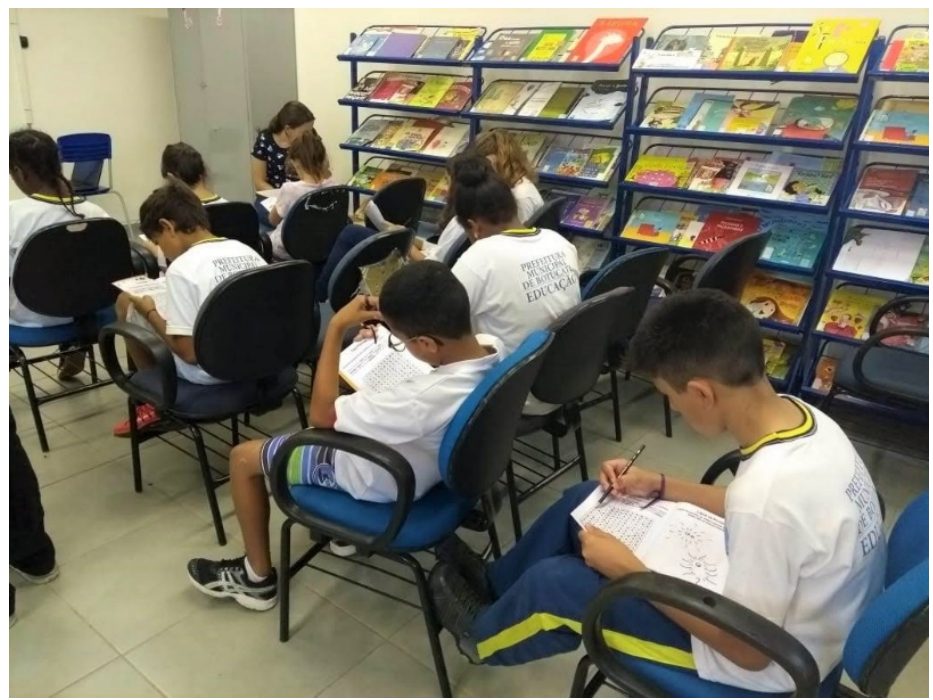

Figura 5. Alunos realizando as atividaddes do material educativo impresso ao final da gincana.

\section{RESULTADOS E DISCUSSÃO}

Houve uma ampla participação das crianças e professores nas atividades desenvolvidas. Foram atendidas 22 turmas, sendo cinco turmas do primeiro ano, cinco do segundo ano, quatro do terceiro ano, quatro do quarto ano e quatro do quinto ano. As faixas etárias das crianças envolvidas no projeto eram de 5 a 11 anos, totalizando a participação de 491 crianças.

Durante a gincana, os membros organizadores registraram as respostas das crianças frente a duas atividades: posicionamento dos alimentos na geladeira e jogo de associações, no intuito de avaliar a porcentagem de erro dos alunos. Isso refletiria, portanto, o quanto eles teriam absorvido das informações durante a palestra interativa. Estes resultados estão dispostos na Tabela 1.

Tabela 1. Porcentagem de erro por turmas durante as atividades do posicionamento dos alimentos na geladeira e do jogo de associações realizados na escola pública de ensino fundamental I Prof. Marins, Botucatu-SP.

\begin{tabular}{ccc}
\hline Turmas & $\begin{array}{c}\text { Posicionamento errado dos alimentos na } \\
\text { geladeira (\%) }\end{array}$ & $\begin{array}{c}\text { Erro no jogo de } \\
\text { associações (\%) }\end{array}$ \\
\hline $\mathbf{1}^{\mathbf{0}}$ & 7,72 & 6,57 \\
$\mathbf{2}^{\mathbf{o}}$ & 9,37 & 1,33 \\
$\mathbf{3}^{\mathbf{o}}$ & 4,85 & 2,19 \\
$\mathbf{4}^{\mathbf{0}}$ & 2,71 & 4,08 \\
$\mathbf{5}^{\mathbf{0}}$ & 12,69 & 5,7 \\
\hline Média & 7,33 & 3,97 \\
total & & \\
\hline
\end{tabular}

O projeto de extensão teve como resultado a transmissão de informações acerca de higiene pessoal e de alimentos, salientando a importância da educação em saúde pública nas escolas. Durante a realização de cada atividade, foi realizada uma avaliação de cada turma, no sentido de identificar os pontos que levaram a curiosidade, discussão, envolvimento e percepção dos hábitos inadequados das crianças. Tal avaliação foi executada por um membro organizador, que preenchia uma planilha de informações sobre o andamento do projeto. 
Durante a aplicação de todas as atividades, as crianças foram instigadas a participar ativamente das atividades, de modo que a equipe fosse capaz de avaliar o nível de conhecimento sobre o assunto.

$\mathrm{Na}$ palestra (etapa inicial da ação), os membros da equipe do projeto realizaram perguntas sobre higiene pessoal, higiene de alimentos, conhecimentos básicos de microbiologia e conservação de alimentos. Neste momento, a cada resposta correta os organizadores elogiavam a atitude do aluno, o que incentiva os demais a responder as próximas perguntas. Quando os alunos respondiam de maneira inadequada, era feito a correção, de modo a fixar o tema abordado naquele momento. Esta interação foi fundamental para a verificação do percentual baixo de erros nas perguntas realizadas.

Após a palestra, os alunos foram divididos em duas turmas e convidados a participar de uma gincana. Esta, como abordado anteriormente, consistiu em três etapas, sendo possível a aplicação da avaliação de conhecimento em duas dessas ações: Brincadeira de posicionar os alimentos na geladeira e o Jogo de associação. Na primeira, os membros da equipe do projeto de extensão utilizaram um cartaz com o desenho de uma geladeira e cartões contendo desenhos de alimentos, os quais os alunos precisavam indicar se eles ficavam dentro ou fora da geladeira.

Tanto a geladeira quanto os cartões com alimentos eram dotados de velcros que permitiam a adesão dos desenhos dos alimentos no cartaz da geladeira. Cada aluno recebeu um cartão e foi convidado a indicar a posição correta dos alimentos na geladeira (tema abordado na palestra). Quando erravam, os membros da equipe do projeto gentilmente realizavam a correção e explicavam o motivo do erro, indicando o local adequado. Nesta ação, a média de erros foi de $7,33 \%$ entre todas as turmas. As porcentagens de erros foram de $7,72 \%, 9,37 \%, 4,85 \%, 2,71 \%$ e $12,69 \%$ para o $1^{\circ}, 2^{\circ}, 3^{\circ}, 4^{\circ}$ e $5^{\circ}$ ano, respectivamente.

$\mathrm{Na}$ segunda atividade da gincana, dois membros da equipe do projeto representaram uma bactéria não patogênica, segurando a imagem de uma bactéria sorrindo, e uma bactéria patogênica, imagem de uma bactéria com "cara de mal". Foram projetadas imagens de alimentos e situaçãos frente às quais os alunos deveriam se direcionar no lado da imagem da bactéria "do bem" ou "do mal". Nesta ação, a média de erros foi de 3,97\% entre todas as turmas. As porcentagens de erros foram de 6,57\%, 1,33\%, 2,19\%, 4,08\% e 5,70\% para o $1^{\circ}$, $2^{\circ}, 3^{\circ}, 4^{\circ}$ e $5^{\circ}$ ano, respectivamente.

Os alunos dos $5^{\circ}$ anos apresentaram maior quantidade de erros na primeira avaliação (posicionamento na geladeira) e no jogo de associação também apresentaram maior quantidade de erros (atrás apenas dos alunos do $1^{\circ}$ ano). Acreditamos que, apesar da maior idade, a ansiedade e pressa para a realização das respostas contribuem para uma maior porcentagem de erros quando comprados com os outros anos. Porém, de qualquer maneira, o resultado foi dentro do esperado.

Essa baixa porcentagem de erro foi devido ao grande interesse por parte das crianças durante a palestra interativa. Os alunos realizaram diversas perguntas relacionadas à manipulação de alimentos e a noções de microbiologia básica. Isso demonstra que a realização da palestra e o momento de conversa entre alunos e organizadores foram essenciais para o entendimento frente ao assunto abordado, os deixando aptos e munidos de conhecimento para desempenhar as atividades subsequentes. Além disso, as turmas que obtiveram a maior porcentagem de erro foram àquelas formadas pelas crianças mais velhas deste estudo, entre 10 e 11 anos, demonstrando a necessidade de reforçar informações acerca de higiene pessoal e de alimentos independentemente da faixa etária.

Atividades que promovem educação em saúde nas escolas são essenciais para a formação de adultos conscientes. Os assuntos apreendidos na infância de maneira lúdica são comprovadamente levados para fase adulta, o que permite que esse conhecimento seja passado de uma pessoa para outra e de geração em geração (13). Diversos projetos de 
extensão universitária usam desta estratégia para levar conhecimento a crianças na rede pública. A transmissão de informação de forma lúdica permite uma maior absorção de conhecimento, uma vez que, por estarem em um momento diferente da rotina escolar, as crianças acabam se permitindo a novos desafios. Tais projetos permitiram com sucesso a promoção de educação em saúde nas escolas, devolvendo para a sociedade os conhecimentos produzidos nas universidades públicas (14-17).

Além dessa questão, é importante salientar que projetos como o apresentado neste artigo demonstram a importância das Universidades para a sociedade, devendo ser incentivadas e curricularizadas junto à graduação, uma vez que colocar pré-profissionais para exercer atividades educativas é fundamental para a construção de uma sociedade mais participativa e menos desigual. Apesar de não constar como plano de avaliação do projeto, ao término deste, foi discutido a importância de sua realização para a formação acadêmica dos voluntários e todos, sem exceção, manifestaram a importância da realização das atividades para suas formações pessoais e profissionais, muitos relatando que foi a atividade mais recompensadora realizada durante a graduação. Deste modo, a execução de atividades extensionistas coloca-se como uma dos alicerces para a formação profissional, sobretudo para graduandos em áreas relacionados com a Saúde Pública.

\section{CONSIDERAÇÕES FINAIS}

Por meio de atividades desenvolvidas em três partes, com palestras, brincadeiras e material didático, foi possível ensinar noções básicas de microbiologia, higiene pessoal e de alimentos. As crianças participaram ativamente, questionando e levantando discussões sobre o assunto, o que demonstrou que a temática era relevante para aquela faixa etária.

Através das atividades lúdicas, foi possível promover educação em saúde de forma prazerosa, no intuito de evitar doenças relacionadas à carência de higiene pessoal e a manipulação incorreta de alimentos. As crianças poderão, portanto, levar o conhecimento adiante e consciêntizar familiares e amigos.

\section{REFERÊNCIAS}

1. Organização Pan-Americana da Saúde. Segurança dos alimentos é responsabilidade de todos [Internet]. Washington: OPAS; 2020 [citado 24 Ago 2020]. Disponível em: https://www.paho.org/bra/index.php?option=com_content\&view $=$ article\&id=5960:seguran ca-dos-alimentos-e-responsabilidade-de

todos\&Itemid=875\#: :text=Estima $\% 2 \mathrm{Dse} \% 20 \mathrm{que} \% 20600 \% 20 \mathrm{milh} \% \mathrm{C} 3 \% \mathrm{~B} 5 \mathrm{es}, \mathrm{mil} \% 20 \mathrm{~m}$ orrem $\% 20 \mathrm{a} \% 20$ cada $\% 20$ ano

2. Brasil. Ministério da Saúde. Doenças transmitidas por alimentos: causas, sintomas, tratamento e prevenção [Internet]. Brasília: Ministério da Saúde; 2020 [citado 24 Ago 2020]. Disponível em: http://www.saude.gov.br/saude-de-a-z/doencas-transmitidas-poralimentos

3. Brasil. Ministério da Saúde. Vigilância epidemiológica de doenças transmitidas por alimentos [Internet]. Brasília: Ministério da Saúde; 2020 [citado 24 Ago 2020]. Disponível em: http://portalarquivos2.saude.gov.br/images/pdf/2019/fevereiro/15/Apresenta----oSurtos-DTA---Fevereiro-2019.pdf

4. Chua JL, Chen M. Barriers and facilitators of reporting foodborne illness. Online J Public Health Inform. 2018;10(1):e101. doi:10.5210/ojphi.v10i1.8754. 
5. Brasil. Ministério da Saúde. Surto de doenças transmitidas por alimentos no Brasil [Internet]. Brasília: Ministério da Saúde; 2018 [citado 10 Maio 2021]. Disponível em: https://portalarquivos2.saude.gov.br/images/pdf/2018/janeiro/17/Apresentacao-SurtosDTA-2018.pdf

6. European Food Safety Authority-EFSA; European Centre for Disease Prevention and Control-ECDC. The european union summary report on trends and sources of zoonoses, zoonotic agents and food-borne outbreaks in 2011. EFSA J. 2013;11(4):3129. doi: 10.2903/j.efsa.2013.3129.

7. Byrd-Bredbenner C, Berning J, Martin-Biggers J, Quick V. Food safety in home kitchens: a synthesis of the literature. Int J Environ Res Public Health. 2013;10(9):4060-85. doi: 10.3390/ijerph10094060.

8. Hou S-I. Health education: theoretical concepts, effective strategies and core competencies. Health Promot Pract. 2014;15(5):619-21.

9. World Health Organization. Health promoting schools [Internet]. Geneva: WHO; 2021 [citado 8 Mar 2021]. Disponível em: https://www.who.int/health-topics/health-promotingschools\#tab=tab_1

10. World Health Organization. One health [Internet]. Geneva: WHO; 2021 [citado 8 Mar 2021]. Disponível em: https://www.who.int/news-room/q-a-detail/one-health

11. Lima GZ. Saúde escolar e educação. São Paulo: Cortez; 1985.

12. Fernandes SR, Park MB. Lembrar-esquecer: trabalhando com memórias infantis. Cad Cedes. 2006;26(68):39-59.

13. Brito AKA, Silva FIC, França NM. Programas de intervenção nas escolas brasileiras: uma contribuição da escola para a educação em saúde. Saude Debate. 2012;36(1):624-32.

14. Joventino ES, Freitas LV, Rogério RF, Lima TM, Dias LMB, Ximenes LB. Jogo da memória como estratégia educativa para prevenção de enteroparasitoses: relato de experiência. Rev RENE. 2009;10(2):141-8.

15. Carolina M, Moraes M, Souza DTB, Fernandes LA, Lima DC. A arte de encantar préescolares com histórias em saúde bucal. Rev Cienc Ext. 2019;15(4):67-81.

16. Silva AVM, Corrêa BS, Carneiro IM, Pinto PR, Almeida AAP, Silva JH. Ações de extensão para promoção da saúde em creche no município de Belo Horizonte, MG. Interagir Pensando Ext. 2016;(22):32-45.

17. Guollo AJ, Lamonega BM, Bastos FVF, Piani SS, Kitamura EA, Souza ALF. PETiscos: relato de ação de extensão sobre educação nutricional de cães e gatos com crianças do ensino fundamental. Rev Cienc Ext. 2019;15(4):82-93.

Recebido em: 05/11/2020 Aceito em: 29/07/2021

Moraes GN, Shing TF, Souza CRR, Danelon GC, Hioka LT, Campanha RT, et al. Educação sanitária com foco na segurança de alimentos: Uma abordagem para crianças. Vet. e Zootec. 2021; v28: 001-010 